\title{
Optical Studies of the Low Velocity Gradient Type Ia Supernovae 2009an and 2009ig
}

\author{
D. K. Sahu, G. C. Anupama, P. Anto, and U. K. Gurugubelli \\ Indian Institute of Astrophysics, Bangalore 560034, India
}

\begin{abstract}
UBVRI photometry and medium resolution spectroscopy of two Type Ia supernovae, SN 2009an and SN 2009ig, are presented. Their $\Delta \mathrm{m}_{15}$ (B) indicate these two SNe marginally deviate from "normal" Type Ia events. Spectroscopically, both SNe belong to the low velocity gradient group. The estimated mass of ${ }^{56} \mathrm{Ni}$ ejected is almost a factor of two different for these two objects, with the estimates being $\sim 0.4 \mathrm{M}_{\odot}$ for $\mathrm{SN} 2009$ an and $\sim 0.8 \mathrm{M}_{\odot}$ for SN 2009ig.
\end{abstract}

Keywords. supernovae: individual (SN 2009an \& SN 2009ig)

\section{Introduction}

SN 2009an was discovered on 2009 February 27.93, and SN 2009ig was discovered on 2009 August 20.48. Both SNe were discovered and classified as Type Ia before maximum. Photometric and spectroscopic observations were made with the $2 \mathrm{~m}$ Himalayan Chandra Telescope of the Indian Astronomical Observatory, Hanle, India, using the Himalayan Faint Object Spectrograph Camera. SN 2009an was observed from 2009 March 01 to 2009 August 03 and SN 2009ig was observed from 2009 August 27 to 2010 February 01.

\section{Results}

\subsection{Light Curves}

Light curves of both SNe Ia have been plotted in Figure 1. SN 2009an reached the maximum in $B$ on JD $2454898.5 \pm 1$ at $14.55 \pm 0.03$ mag. The early decline, $\Delta \mathrm{m}_{15}(\mathrm{~B})=$ $1.514 \pm 132$ is found to be intermediate between the normal SNe Ia and the fast declining SN 1991bg type events. SN 2009ig reached maximum in $B$ band on JD $2455080.8 \pm 0.6$ with a magnitude $13.57 \pm 0.03$. Its decline at $\Delta \mathrm{m}_{15}(\mathrm{~B})=0.95 \pm 0.02$ is slower than that of normal SNe Ia. Both SNe show secondary maxima in the $R$ and $I$ bands.

\subsection{Reddening, Absolute Magnitude, and Mass of ${ }^{56} \mathrm{Ni}$}

Reddening values have been estimated for both SN 2009an and SN 2009ig using the $\mathrm{NaI} \mathrm{D}$ line seen in the spectrum close to maximum light, as well as various empirical relations assuming that all normal SNe Ia have similar colors at different phases. The total reddening $\mathrm{E}(\mathrm{B}-\mathrm{V})_{\text {total }}$ estimates are 0.11 and 0.10 for SN 2009an and SN 2009ig, respectively. The absolute magnitudes have been estimated using the various versions of relations between peak absolute magnitude and $\Delta \mathrm{m}_{15}(\mathrm{~B})$. An average of peak absolute magnitude in $B$ band for SN 2009an is $-18.84 \pm 0.16$ and for SN 2009ig is $-19.43 \pm 0.06$, indicating SN 2009an is underluminous and SN 2009ig is overluminous compared to normal SNe Ia.

The monochromatic $U B V R I$ fluxes have been integrated over the wavelength range $3100 \AA$ to $10600 \AA$ to get the bolometric flux. With a rise time of 20 days and estimated 
peak bolometric flux, the mass of ${ }^{56} \mathrm{Ni}$ synthesized in the explosion is estimated using Arnett's Rule (Arnett 1982) as $\sim 0.4 \mathrm{M}_{\odot}$ for SN 2009an and $0.8 \mathrm{M}_{\odot}$ for SN 2009ig.

\subsection{Spectral evolution}

Early phase spectra of both SNe show blue continua with a well developed P-Cygni profile of the Si II $\lambda 6355$ line. Other features include Ca II H\&K, Mg II $\lambda 4481$, well developed W shape S II lines and weak Ca II NIR triplet, with two components corresponding to low and high velocities. In the immediate post maximum phase the absorption strength of intermediate mass elements increases.

In the nebular phase, spectra of SN 2009an and SN 2009ig are dominated by forbidden lines of singly and doubly ionized Co and Fe. The spectral evolution of SN 2009an is very similar to transitional Type Ia supernova SN 2004eo (Pastorello et al. 2007), while the spectral evolution of SN 2009ig is very similar to SN 1999aa (Garavini et al. 2004).

The expansion velocity of SN 2009an starts with $\sim 14,500 \mathrm{~km} \mathrm{~s}^{-1}$ and levels off at $\sim 10,000 \mathrm{~km} \mathrm{~s}^{-1}$, whereas the expansion velocity of SN 2009ig remains high $(\sim 12,500$ $\mathrm{km} \mathrm{s}^{-1}$ ) till the last day of observations. The velocity gradient in SN 2009an is found to be $\sim 60$, which is just at the upper limit of the low velocity gradient class, while SN 2009ig falls in the low velocity gradient category of Benetti's classification (2005).

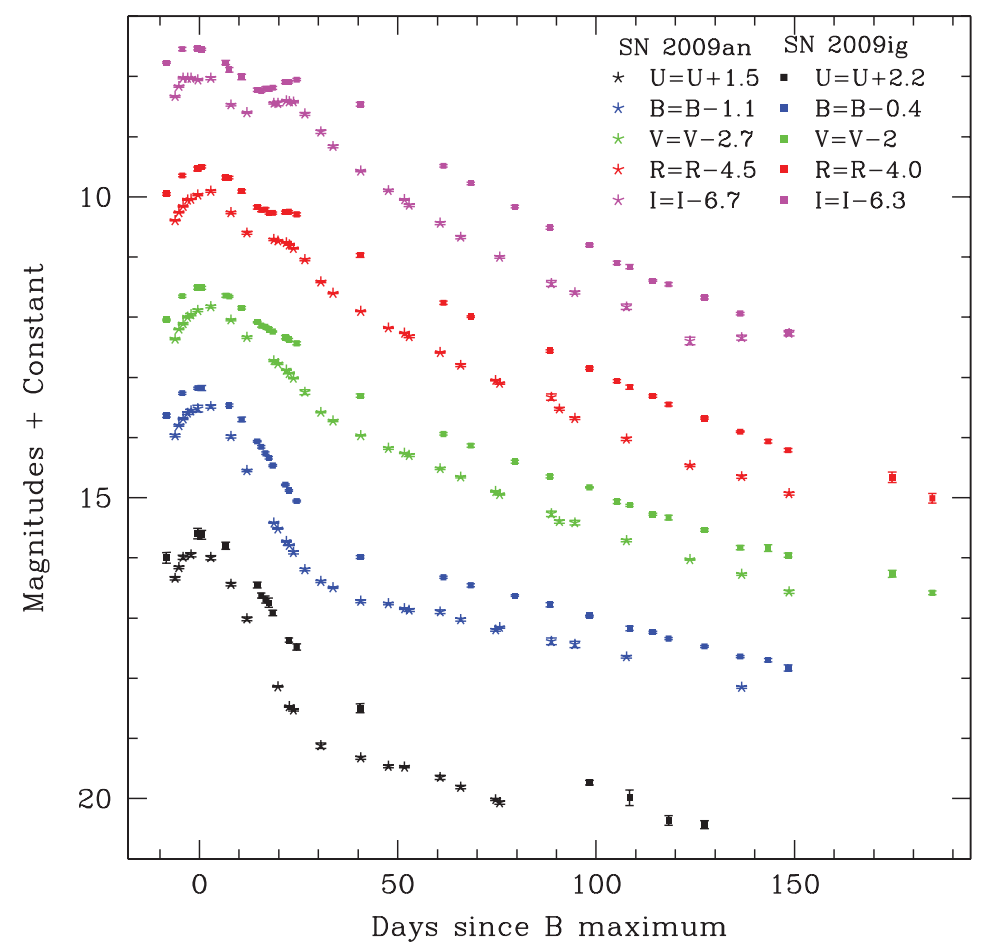

Figure 1. Light curves of SNe 2009an and 2009ig 

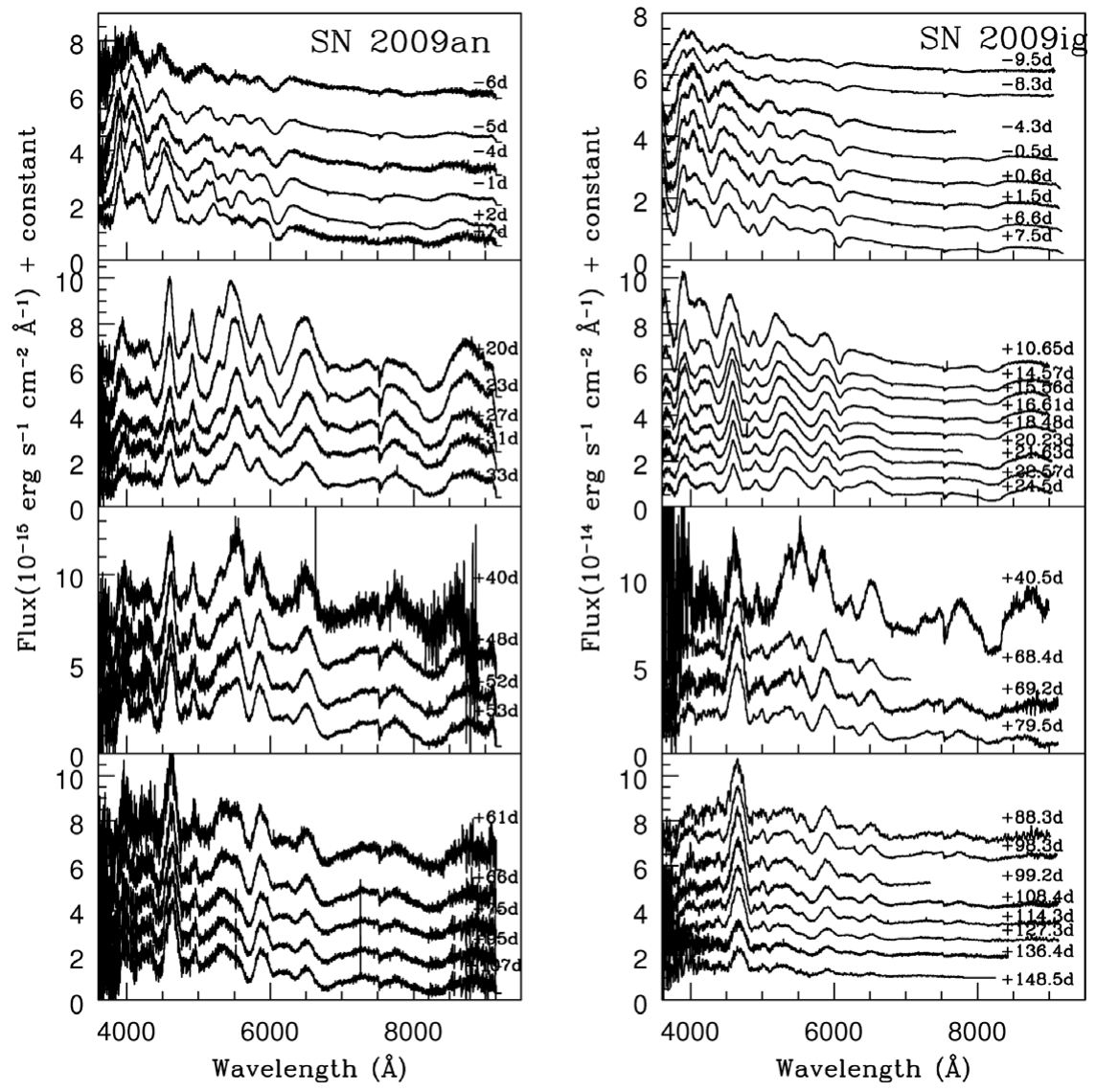

Figure 2. Spectral evolution of SNe 2009an and 2009ig

\section{References}

Arnett, W. D. 1982, 253, 785

Benetti, S. et al. 2005, APJ, 623, 1011

Garavini, G. et al. 2004, AJ, 128, 387

Pastorello, A. et al. 2007, MNRAS, 276, 1301 